\title{
Genetic differences in the serum proteome of horses, donkeys and mules are detectable by protein profiling
}

\author{
Andrea Henze $^{1 *}$, Franziska Aumer ${ }^{1,2}$, Arthur Grabner ${ }^{2}$, Jens Raila ${ }^{1}$ and Florian J. Schweigert ${ }^{1}$ \\ ${ }^{1}$ Department of Physiology and Pathophysiology of Nutrition, Institute of Nutritional Science, University of Potsdam, \\ Arthur-Scheunert-Allee 114-116, 14558 Nuthetal, Potsdam, Germany \\ ${ }^{2}$ Equine Clinic, Department of Veterinary Medicine, Freie Universität Berlin, Berlin, Germany
}

(Received 15 October 2010 - Revised 13 January 2011 - Accepted 28 January 2011)

\begin{abstract}
Although horses and donkeys belong to the same genus, their genetic characteristics probably result in specific proteomes and posttranslational modifications (PTM) of proteins. Since PTM can alter protein properties, specific PTM may contribute to species-specific characteristics. Therefore, the aim of the present study was to analyse differences in serum protein profiles of horses and donkeys as well as mules, which combine the genetic backgrounds of both species. Additionally, changes in PTM of the protein transthyretin (TTR) were analysed. Serum protein profiles of each species (five animals per species) were determined using strong anion exchanger ProteinChips $^{\circledR}$ (Bio-Rad, Munich, Germany) in combination with surface-enhanced laser desorption ionisation-time of flight MS. The PTM of TTR were analysed subsequently by immunoprecipitation in combination with matrix-assisted laser desorption ionisation-time of flight MS. Protein profiling revealed species-specific differences in the proteome, with some protein peaks present in all three species as well as protein peaks that were unique for donkeys and mules, horses and mules or for horses alone. The molecular weight of TTR of horses and donkeys differed by $30 \mathrm{Da}$, and both species revealed several modified forms of TTR besides the native form. The mass spectra of mules represented a merging of TTR spectra of horses and donkeys. In summary, the present study indicated that there are substantial differences in the proteome of horses and donkeys. Additionally, the results probably indicate that the proteome of mules reveal a higher similarity to donkeys than to horses.
\end{abstract}

\section{Key words: Mass spectrometry: Post-translational modifications: Proteome: Species differences}

Horses and donkeys both belong to the genus Equus, however they represent individual species with distinct genetic characteristics. These characteristics can result in a speciesspecific variability of the proteome ranging from shifts in molecular weight due to amino acid substitutions, deletions or insertions to the absence or presence of proteins or protein complexes. In addition, the genetic predisposed differences of the proteome may also influence the potential of posttranslational modification (PTM) of proteins by providing or rejecting modification partners such as phosphorylation, oxidation or glycosylation. Since PTM can alter protein properties such as transport function, protein-protein interaction and antioxidant capacity, species-specific PTM may contribute to species-specific characteristics especially with regard to metabolism $^{(1,2)}$.

Therefore, the aim of the present study was to analyse differences in serum protein patterns of horses and donkeys in order to obtain insight into possible metabolism-related differences of these closely related species. Furthermore, changes in PTM patterns were specifically analysed for the visceral protein transthyretin (TTR). TTR is a homotetrameric protein, which is directly involved in the transport of thyroxin and indirectly in the transport of vitamin $\mathrm{A}^{(3)}$. TTR represents an optimal model for studies on PTM, since it possesses a single cysteine residue that is not involved in any disulphide bonds and that can be extensively modified. Among others, dihydroxylation (mass shift of $30 \mathrm{Da}$ ), sulfonation $(80 \mathrm{Da})$, cysteinylation $(120 \mathrm{Da})$ as well as the addition of cysteinylglycine $(175 \mathrm{Da})$ as PTM of TTR have been described ${ }^{(4,5)}$.

Serum analyses were aslo performed in mules. Since mules originate from the cross-breeding of female horses and male donkeys, they should theoretically combine the genetic backgrounds of both species. Therefore, it may be reasonable that the serum protein patterns and PTM of mules represent individual characteristics from both horses and donkeys.

\section{Materials and methods}

Serum samples of five horses (three females and two males), five donkeys (one female and four males) and five mules

Abbreviations: MALDI-TOF-MS, matrix-assisted laser desorption ionisation-time of flight MS; PTM, post-translational modification; TTR, transthyretin. 
(three females and two males) were obtained from Equine Clinic, Department of Veterinary Medicine, Freie Universität, Berlin, Germany. Serum was collected during routine examination from the jugularis vein, centrifuged and stored at $-80^{\circ} \mathrm{C}$ until analysis.

As samples were collected from the species as part of a routine clinical evaluation at the clinic, approval from the university's animal care and use committee was not required. Client permission was obtained for the use of the samples. For protein pattern profiling, serum samples were prepared using strong anion exchanger ProteinChips ${ }^{\circledR}$ (Bio-Rad, Munich, Germany) according to the manufacturer's instruction. ProteinChips ${ }^{\circledR}$ (Bio-Rad) were subsequently analysed using surface-enhanced laser desorption ionisation-time of flight MS (Ciphergen Biosystems, Fremont, CA, USA) as reported previously $^{(6)}$. Protein patterns were evaluated statistically using Ciphergen Peak Software version 3.2 (Ciphergen Biosystems, Fremont, CA, USA). A two-tailed $t$ test for statistical analysis of differences in the molecular weight of protein peak clusters between species was used (Biomarker Wizard software; Ciphergen Biosystems). The level of significance was assigned at $P<0.02$ for a more demanding statistical analysis as suggested in the Biomarker Wizard software instructions.

For determination of PTM, serum TTR was enriched by immunoprecipitation as described before and analysed with matrix-assisted laser desorption ionisation-time of flight MS (MALDI-TOF-MS; Bruker Daltonics, Bremen, Germany) $)^{(7)}$. To identify native TTR, representative immunoprecipitates of each species were treated with dithiothreitol and analysed by MALDI-TOF-MS as described previously ${ }^{(8)}$.

\section{Results}

The results of the protein profiling within the mass range from 3 to $30 \mathrm{kDa}$ are presented in Table 1 . The molecular weights of the detected peaks tended to be lower in horses than in mules or donkeys. However, if the deviation remained within the tolerable range, Ciphergen Peak Software assigned the proteins to the same protein clusters as for donkeys and mules. A total of thirty-one protein peak clusters could be detected, of which fifteen (peaks 1, 3, 5, 13-19, 21, 23, 25, 26 and 30) were present in all three species. Additionally, seven peaks could be determined only in donkeys and mules (2, 7, 20, 24, 27, 28 and 31), while three peaks were present only in horses and mules (4, 8 and 9). Furthermore, there were six peaks, which were present only in horses (6, 10-12, 22 and 29). In contrast, no peaks could be detected, which were unique for donkeys or UNIQUE FOR mules.

Representative spectra of the analysis of TTR as a model for determination of differences in PTM patterns are given in Fig. 1(A). The analysis revealed six TTR peaks in horses (peaks $\mathrm{a}-\mathrm{f}$ ) as well as in donkeys (peaks A-F), which were arranged in identical patterns in both species (Fig. 1(A)). The corresponding peaks of horses and donkeys differed by approximately $30 \mathrm{Da}$, and the exact molecular weight of each peak is given in the legend of Fig. 1.

To identify the native TTR, the immunoprecipitates were treated with dithiothreitol ${ }^{(8)}$. The subsequent MALDI-TOF-MS
Table 1. Molecular weights $(\mathrm{Da})$ of protein peaks detected by ProteinChip analysis with surface-enhanced laser desorption ionisationtime of flight MS in the serum of horses, mules and donkeys

(Mean values and standard deviations)

\begin{tabular}{|c|c|c|c|c|c|c|}
\hline \multirow[b]{2}{*}{ PC } & \multicolumn{2}{|c|}{ Horses } & \multicolumn{2}{|c|}{ Mules } & \multicolumn{2}{|c|}{ Donkeys } \\
\hline & Mean & SD & Mean & SD & Mean & SD \\
\hline 1 & $4171^{*} \dagger$ & 13 & 4196 & 7 & 4197 & 12 \\
\hline 2 & $\mathrm{n} / \mathrm{d}$ & & 4287 & 7 & 4287 & 6 \\
\hline 3 & $4540^{*} \dagger$ & 5 & 4567 & 7 & 4563 & 6 \\
\hline 4 & $4723 \dagger$ & 5 & 4749 & 8 & $\mathrm{n} / \mathrm{d}$ & \\
\hline 5 & 4883 & 9 & 4880 & 8 & 4881 & 7 \\
\hline 6 & 5096 & 55 & $\mathrm{n} / \mathrm{d}$ & & $n / d$ & \\
\hline 7 & $n / d$ & & 5451 & 10 & 5454 & 6 \\
\hline 8 & $5548 \dagger$ & 8 & 5581 & 9 & $n / d$ & \\
\hline 9 & $5759 \dagger$ & 9 & 5785 & 7 & $n / d$ & \\
\hline 10 & 7016 & 10 & $n / d$ & & $\mathrm{n} / \mathrm{d}$ & \\
\hline 11 & 7133 & 10 & $\mathrm{n} / \mathrm{d}$ & & $\mathrm{n} / \mathrm{d}$ & \\
\hline 12 & 7336 & 6 & $n / d$ & & $n / d$ & \\
\hline 13 & $7666 \dagger$ & 8 & 7593 & 33 & 7639 & 27 \\
\hline 14 & $8065^{\star} \dagger$ & 9 & $8093^{*}$ & 12 & 8125 & 10 \\
\hline 15 & $8258^{*} \dagger$ & 15 & 8299 & 7 & 8299 & 9 \\
\hline 16 & $8514^{*} \dagger$ & 8 & 8543 & 11 & 8541 & 9 \\
\hline 17 & $8785^{\star} \dagger$ & 8 & $8808^{*}$ & 14 & 8768 & 9 \\
\hline 18 & $9054^{*} \dagger$ & 2 & 9090 & 8 & 9084 & 12 \\
\hline 19 & $9419^{*}$ & 11 & 9452 & 25 & 9462 & 15 \\
\hline 20 & $n / d$ & & 11483 & 12 & 11495 & 16 \\
\hline 21 & 12621 & 13 & 12548 & 100 & 12589 & 82 \\
\hline 22 & 13112 & 47 & $n / d$ & & $n / d$ & \\
\hline 23 & 13905 & 10 & 13929 & 17 & 13923 & 26 \\
\hline 24 & $n / d$ & & $14868^{*}$ & 13 & 14870 & 23 \\
\hline 25 & 17238 & 18 & 17258 & 28 & 17261 & 16 \\
\hline 26 & $21860 \dagger$ & 19 & 21906 & 28 & 21874 & 17 \\
\hline 27 & $n / d$ & & $22975^{*}$ & 20 & 22908 & 17 \\
\hline 28 & $n / d$ & & $24926^{*}$ & 84 & 25112 & 106 \\
\hline 29 & 26142 & 41 & $n / d$ & & $n / d$ & \\
\hline 30 & $27590^{*} \dagger$ & 27 & 27699 & 28 & 27685 & 20 \\
\hline 31 & $n / d$ & & $29612^{\star}$ & 107 & 29843 & 108 \\
\hline
\end{tabular}

PC, protein cluster; MW, molecular weight; $\mathrm{n} / \mathrm{d}$, not detected.

* Mean values were significantly different from those of donkeys $(P<0.02)$. Although there was a significant difference, the peptides and proteins were assigned to a protein cluster according to Biomarker Wizard software (Ciphergen Biosystems, Fremont, CA, USA).

$\dagger$ Mean values were significantly different from those of mules $(P<0.02)$. Although there was a significant difference, the peptides and proteins were assigned to a protein cluster according to Biomarker Wizard software (Ciphergen Biosystems).

analysis (Fig. 1(B)) revealed single dominant peaks of $13797 \mathrm{Da}$ in horses (corresponding to peak a) and $13827 \mathrm{Da}$ in donkeys (corresponding to peak A), which were assigned to the native TTR.

Besides the native TTR, the other TTR variants differed by approximately 30, 80, 120, 140 and $175 \mathrm{Da}$ in both horses and donkeys. As a result, the modifications of 120 and $175 \mathrm{Da}$ represented the most dominant forms in non-reduced TTR in both species. According to the literature, the modification of $30 \mathrm{Da}$ was assigned to dihydroxylated TTR (peaks b and B), 80 Da to sulfonated TTR (peaks c and C), 120 Da to cysteinylated TTR (peaks d and D) and 175 Da to cysteinylglycinated TTR (peaks $\mathrm{f}$ and $\mathrm{F})^{(4)}$. However, the modification with a mass increase of $140 \mathrm{Da}$ (peaks e and E) remains unknown. Additionally, the spectra of some animals revealed a mass shift by approximately $-42 \mathrm{Da}$ (indicated as * in the spectra of donkey in Fig. 1(A)), which may represent a conversion of arginine to ornithine according to the Delta Mass Database $^{(9)}$. 


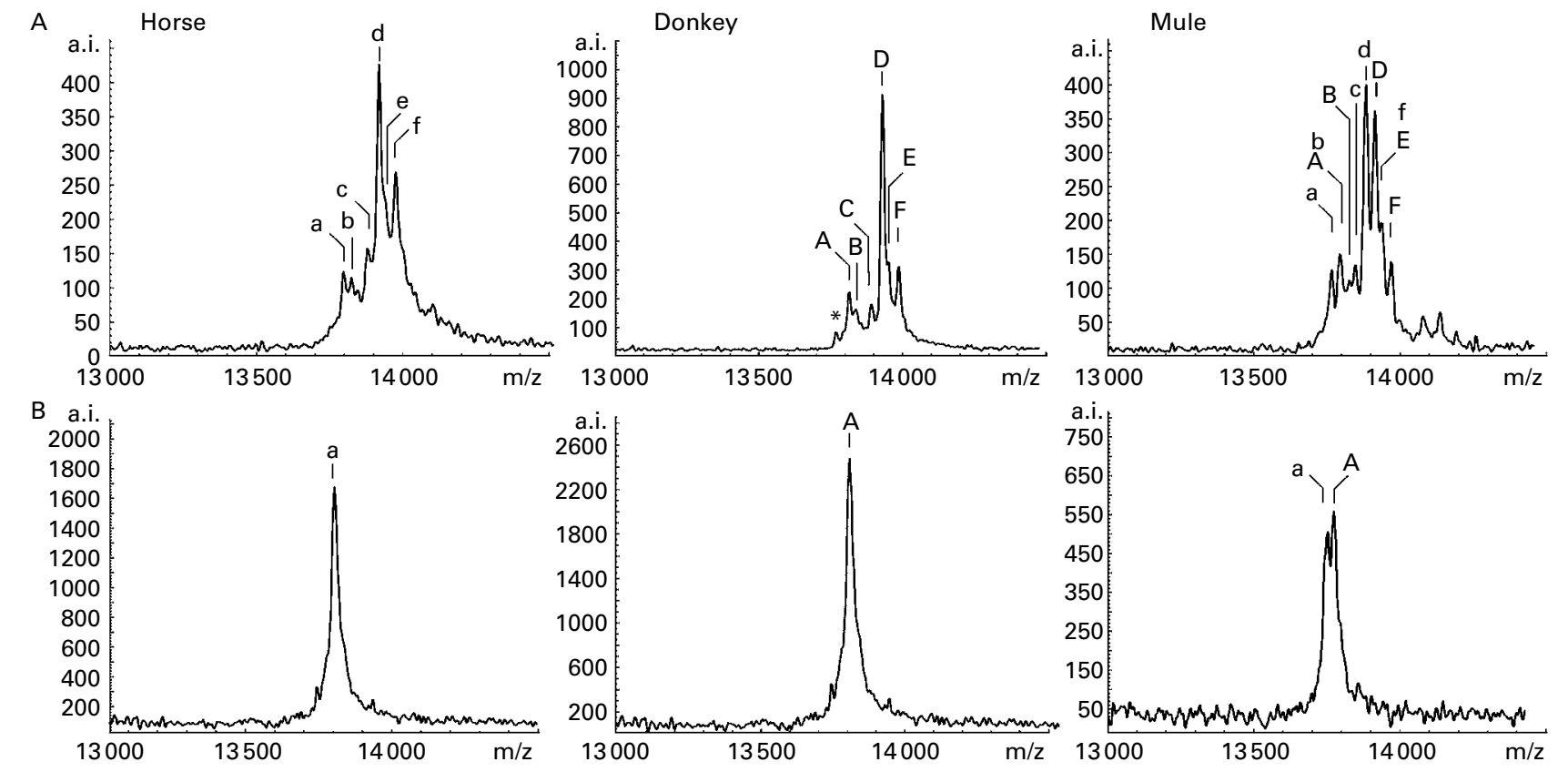

Fig. 1. Representative matrix-assisted laser desorption ionisation-time of flight MS spectra from transthyretin (TTR) of horses, donkeys and mules immunoprecipitated from $(A)$ serum and $(B)$ after dithiothreitol treatment. The TTR variants revealed the following molecular weights: a, $13797 \mathrm{Da}( \pm 4)$; b, 13839 Da $( \pm 7)$; c, $13878 \mathrm{Da}( \pm 4)$; d, $13916 \mathrm{Da}( \pm 4)$; e, $13937 \mathrm{Da}( \pm 3) ; f, 13966 \mathrm{Da}( \pm 16) ; \mathrm{A}, 13827 \mathrm{Da}( \pm 4) ; \mathrm{B}, 13846 \mathrm{Da}( \pm 7) ; \mathrm{C}, 13906 \mathrm{Da}( \pm 4) ; \mathrm{D}, 13946 \mathrm{Da}( \pm 4)$; E, $13966 \mathrm{Da}( \pm 4) ; \mathrm{F}, 14002 \mathrm{Da}( \pm 4)$. The * indicates a TTR variant with $-42 \mathrm{Da}$, which was present in some of the analysed samples; a.i., arbitrary index.

Since mules represent one TTR allele from horses and one TTR allele from donkeys, the mass spectra of mules represented a merging of mass spectra from horses and donkeys. Therefore, horse-specific (peaks a, c and d) as well as donkey-specific peaks (peaks B, D and F) could be identified. However, due to the slight mass shift between horses and donkeys, not all TTR variants of horses and donkeys could be recovered in mules. This is most probably due either to the domination by other peaks (e.g. donkeys' TTR peak $\mathrm{C}$ is dominated by horses' TTR peak d) or to identical molecular weights (e.g. horses' TTR peak b and donkeys' TTR peak A) and precluded a definite identification.

\section{Discussion}

The high degree of agreement of protein patterns in the serum of horses and donkeys emphasises the high phylogenetic relationship of these two species. However, protein profiling does also reveal that despite the phylogenetic similarity, the genetic differences account for significant differences in the serum proteome of these species. Furthermore, mules theoretically combine the complete genetic backgrounds of horses and donkeys. The parallel existence of a horse allele and a donkey allele could be clearly shown in the TTR spectra of all three species, indicating that mules are genetic overlays of horses and donkeys. However, protein profiling revealed a higher similarity between mules and donkeys at the protein level, and it also became apparent that some horse-specific proteins are not detectable in mules. This may indicate that the expression of several horse-specific genes is suppressed in mules and may account for the higher robustness of mules in comparison with horses. The proteome reflects the metabolism of individuals and therefore also differences in the metabolism between different individuals or, as in the present study, between different species. These differences in metabolism may be attributed to numerous factors. However, one factor of enormous importance in this context is nutrition. Every nutritional process relies on the interplay of a variety of proteins ${ }^{(10)}$. Furthermore, in terms of nutrition, mules more closely resemble donkeys than horses (Robinson and Slade). Therefore, the differences and analogies in proteomes in the present study may be mainly attributed to differences in species-specific food processing, as already demonstrated for copper sensitivity of Cambridge sheep and North Ronaldsay sheep ${ }^{(11)}$. However, further studies in combination with feeding experiments are necessary for a detailed examination of the influence of nutrition on protein profiles.

An interesting example for the influence of nutrition on the proteome is the protein TTR. In this context, it has been demonstrated that the biological values of proteins in the diet determine the PTM pattern of TTR in the serum of rats $^{(12)}$. This characteristic of TTR is probably due to the free cysteine residue, which is, as with every free cysteine residue, part of the endogenous extracellular antioxidant defence system $^{(13,14)}$. The comparison of the PTM pattern of TTR from horses and donkeys did not reveal a significant difference. However, the interpretation of TTR variants from mules is difficult due to the genetic overlay, but it seems very likely that there are no differences in PTM patterns in comparison with either horses or donkeys. Moreover, all three species revealed PTM comparable with species with a free cysteine residue at position 10 of the TTR monomers 
(humans and rats) ${ }^{(4)}$. These results strongly indicate the existence of a free cysteine residue also in the TTR of horses, donkeys and mules, which may be important in the endogenous extracellular antioxidant defence system of all three species. Furthermore, a higher degree of modification of the free cysteine residue may indicate oxidative stress in horses, donkeys and mules. The present results may indicate an endogenous antioxidant defence system of horses, donkeys and mules comparable with humans and rats. However, due to the distinct differences in protein patterns of the three species, further analyses are needed to evaluate PTM of the whole proteomes.

\section{Acknowledgements}

A. G. and F. J. S. contributed to the conception and design of the study. F. A. and A. H. contributed to the acquisition and interpretation of the data, and drafted of the manuscript. A. H., J. R., A. G. and F. J. S. provided the significant academic advice and consultation through interpretation of the data and writing of the manuscript. All authors contributed to the revision of the manuscript and approved the final version. There is no conflict of interest for any of the authors. F. A. is now a member of the Klinik für Pferde of the Ludwig-MaximiliansUniversität, München, Germany.

\section{References}

1. Zybailov BL \& Washburn MP (2007) Mass spectrometrybased methods of proteome analysis. In Proteins, pp. 697-739 [RA Meyers, editor]. Weinheim: Wiley-VCH.

2. Jungblut PR, Holzhutter HG, Apweiler R, et al. (2008) The speciation of the proteome. Chem Cent J 2, 16
3. Ingenbleek Y \& Young V (1994) Transthyretin (prealbumin) in health and disease: nutritional implications. Annu Rev Nutr 14, 495-533.

4. Terazaki H, Ando Y, Suhr O, et al. (1998) Post-translational modification of transthyretin in plasma. Biochem Biophys Res Commun 249, 26-30.

5. Citron M, Diehl TS, Gordon G, et al. (1996) Evidence that the 42- and 40-amino acid forms of amyloid beta protein are generated from the beta-amyloid precursor protein by different protease activities. Proc Natl Acad Sci U S A 93, $13170-13175$.

6. Schweigert FJ, Gericke B, Wolfram W, et al. (2006) Peptide and protein profiles in serum and follicular fluid of women undergoing IVF. Hum Reprod 21, 2960-2968.

7. Gericke B, Raila J, Deja M, et al. (2007) Alteration of transthyretin microheterogeneity in serum of multiple trauma patients. Biomark Insights 2, 1-8.

8. Gericke B, Raila J, Sehouli J, et al. (2005) Microheterogeneity of transthyretin in serum and ascitic fluid of ovarian cancer patients. BMC Cancer 5, 133

9. Witze ES, Old WM, Resing KA, et al. (2007) Mapping protein post-translational modifications with mass spectrometry. Nat Methods 4, 798-806.

10. Fuchs D, Winkelmann I, Johnson IT, et al. (2005) Proteomics in nutrition research: principles, technologies and applications. Br J Nutr 94, 302-314.

11. Simpson DM, Mobasheri A, Haywood S, et al. (2006) A proteomics study of the response of North Ronaldsay sheep to copper challenge. BMC Vet Res $\mathbf{2}, 36$.

12. Henze A, Rohn S, Gericke B, et al. (2008) Structural modifications of serum transthyretin in rats during protein-energy malnutrition. Rapid Commun Mass Spectrom 22, 3270-3274

13. Halliwell B \& Gutteridge JM (1990) The antioxidants of human extracellular fluids. Arch Biochem Biophys 280, 1-8.

14. Zhang Q \& Kelly JW (2003) Cys10 mixed disulfides make transthyretin more amyloidogenic under mildly acidic conditions. Biochemistry 42, 8756-8761. 